\title{
An unusual case of vaccine-associated paralytic poliomyelitis
}

\author{
S Desai MD FRCPC ${ }^{1}$, T Diener MBChB MMed (Com Health) MPA DTM MFTM RCPS (Glasgow) ${ }^{2}$, BJ-K Tan MD FRCPC ${ }^{3}$, \\ NJ Lowry MD FRCPC ${ }^{4}$, C Talukdar MBBS DCH (Glasgow) FRCPC DABP (USA) FAAP5, \\ WM Chrusch MD FRCPC 6 , S Wiebe MD FRCPC ${ }^{7}$,
}

\begin{abstract}
S Desai, T Diener, NJ Lowry, et al. An unusual case of vaccineassociated paralytic poliomyelitis. Can J Infect Dis Med Microbiol 2014;25(4):227-228.
\end{abstract}

The present article reports a case involving an immunocompetent, previously well child who, despite two previous doses of inactivated poliovirus vaccine, developed severe flaccid paralysis consistent with polio after receiving oral polio vaccine.

Key Words: MRI findings; Oral polio vaccine; Vaccine-associated poliomyelitis

\section{CASE PRESENTATION}

A previously well child who was almost one year of age, born in Canada to Chinese parents, presented to his pediatrician with acute flaccid paralysis of his left leg. He had received his first two sets of immunizations at his local public health unit. This included: diphtheria, tetanus and acellular pertussis (DTP); inactivated poliomyelitis vaccine (IPV); and Haemophilus influenzae type b (Pentacel; Sanofi-Pasteur, Canada) and Prevnar (Pfizer, Canada). He did not experience any adverse events associated with either of these immunizations. At five months of age, the child and his family went to China to visit friends and relatives. At six months of age, his parents continued his immunizations according to the local immunization schedule. He received a dose of DTP and oral poliovirus vaccine (OPV) and, one week later, received a dose of hepatitis $B$ vaccine. Approximately three weeks after immunization with DTP and OPV, he presented to the local children's hospital with fever and limb weakness. On history, he had not experienced any preceding cough or rhinorrhea, nor did he have diarrhea, vomiting or rashes. He had no known sick contacts. His parents had noted a regression in his motor skills, specifically in his ability to stand with support and also difficulty with using his upper extremities.

In China, he was diagnosed with Guillain-Barré syndrome (GBS) based on the presentation of acute flaccid paralysis that was assessed to be symmetrical in nature. Further investigations at the time of presentation included a normal immune work-up, a cerebrospinal fluid white blood cell count of $12 \times 10^{6} / \mathrm{L}$ with a protein level of $1.43 \mathrm{~g} / \mathrm{L}$ and glucose level within normal limits. Cerebrospinal fluid viral culture and polymerase chain reaction were not conducted. Magnetic resonance imaging (MRI) performed in China showed enhancement of the cauda equina.

He was treated with a course of intravenous immunoglobulin ( $1 \mathrm{~g} / \mathrm{kg} /$ day for three days) and steroids (tapering $5 \mathrm{mg}, 3 \mathrm{mg}$ and $1.5 \mathrm{mg}$ over six days). He was discharged from hospital within two weeks, having experienced an improvement in his upper extremity

\section{Un cas inhabituel de poliomyélite paralytique associée au vaccin}

Le présent article rend compte du cas d'un enfant immunocompétent auparavant en santé qui, malgré deux doses antérieures de vaccin inactivé contre le poliovirus, a contracté une grave paralysie flasque qui cadrait avec la polio après s'être fait administrer le vaccin antipoliomyélitique oral. strength and improvement most notably of his right leg. Two weeks after discharge, his family returned to Canada.

On return to Canada, he was seen by his pediatrician. On examination, he had left leg weakness with absent deep tendon reflexes and his sensorium was intact. Urinary stream and anal sphincter tone were normal. His pediatrician recommended further follow-up with a pediatric neurologist and rehabilitation services to help with muscle strength. He was seen at the regional Children's Hospital. At that time, the physical findings were confirmed, a diagnosis of post-GBS was given and further rehabilitation was suggested.

Six weeks later, the child was seen again by a pediatric neurologist and was noted to have asymmetric weakness of his left leg. Because this did not appear to be improving, further treatment and investigations were initiated. The child received three additional days of intravenous immunoglobulin $(0.4 \mathrm{mg} / \mathrm{kg} / \mathrm{day}$ for five days $)$. Subsequently, he underwent electromyography and MRI of his spinal cord. The electromyography showed denervation to muscles of the left leg with an absent peroneal nerve response, tiny motor responses with very slow conduction of the tibial nerve and fibrillation potentials suggesting active denervation in the muscles tested.

Repeat MRI revealed bilateral symmetric increased signal within the anterior horns of the spinal cord gray matter, with abnormal signal identified in two locations (C2 through C5 and T10 through T12) (Figure 1). The multifocal increased signal within the ventral horns of the spinal cord gray matter was highly suggestive of poliomyelitis.

Further investigation revealed his immunization history in China. Follow-up of stool culture showed no nonpolio enteroviral growth and no presence of polio type 1 or type 2 viruses, but did show presence of poliovirus type 3. At the national laboratory in China, the strain was sequenced and was found to be a Sabin poliovirus type 3 strain with 99.7\% homology with the parent OPV strain.

In Canada, further testing included repeat stool culture and throat swab, both negative for poliovirus, polio antibody titres of $<1: 8$ for

${ }^{1}$ Vaccine Preventable Diseases, Centre for Immunization and Respiratory Infectious Diseases, Public Health Agency of Canada, Ottawa, Ontario;

${ }^{2}$ Population and Public Health Services, Regina Qu'Appelle Health Region, Regina; ${ }^{3}$ Infectious Diseases, Department of Pediatrics; ${ }^{4}$ Neurology,

Department of Pediatrics, University of Saskatchewan, Saskatoon; ${ }^{5}$ Pediatrician, Regina; ${ }^{6}$ Physical Medicine and Rehabilitation, Regina

Qu'Appelle Health Region, Regina; ${ }^{7}$ Radiology, Saskatoon Health Region, Saskatoon, Saskatchewan

Correspondence: Dr S Desai, Public Health Agency of Canada, 180 Queen Street West, Toronto, Ontario M5V 3L7.

Telephone 416-952-2238, e-mail shalini.desai@phac-aspc.gc.ca

OPEN ACCESS

This open-access article is distributed under the terms of the Creative Commons Attribution Non-Commercial License (CC BY-NC) (http:// creativecommons.org/licenses/by-nc/4.0/), which permits reuse, distribution and reproduction of the article, provided that the original work is properly cited and the reuse is restricted to noncommercial purposes. For commercial reuse, contact support@pulsus.com 


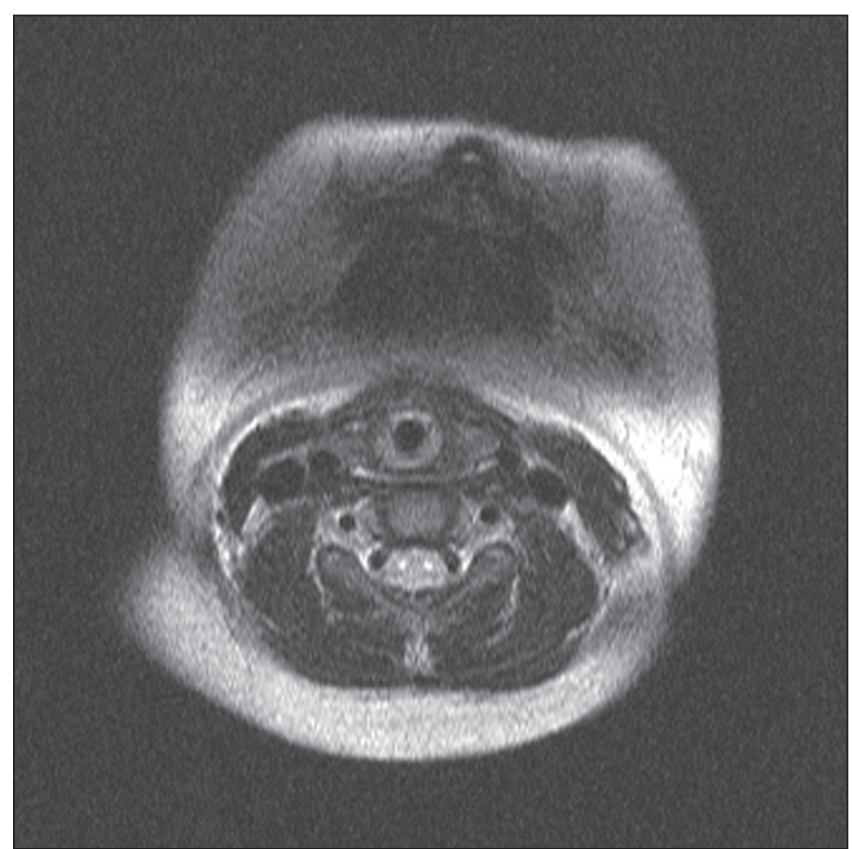

Figure 1) Magnetic resonance image obtained in Canada showing bilateral symmetric increased signal within the anterior horn

poliovirus 1, 1:32 for poliovirus 2 and 1:128 for poliovirus 3 . Immunoglobulin subclass analysis showed no abnormalities and an HIV test was negative. Repeat polio serology was performed seven months after the first set and showed a 1:8 titre for poliovirus 3 .

This suspected case of vaccine-associated paralytic poliomyelitis (VAPP) was reviewed by an expert committee in vaccine-related adverse events - the Advisory Committee Causality Assessment (ACCA) - and was determined to be a probable case of VAPP.

\section{DISCUSSION}

In 1955, the IPV was introduced, with the subsequent introduction of the trivalent OPV being introduced in 1962. The oral vaccine has been used as the major vaccine in the fight to eradicate polio. As countries have been able to control the incidence of polio, the issue of VAPP has represented a larger burden of disease than wild-type virus (1). In light of this information, countries such as Canada have switched from OPV to IPV. Canada was certified polio free in 1994 (2). IPV for routine immunizations was recommended in 1995/1996 and a switch to this occurred across the country (1). The most recent case of VAPP in Canada was reported in 1995 (3). In other countries, such as China, OPV has continued to be used for routine immunizations. China was certified free of wild-type poliovirus in 2000, with their last endemic case occurring in 1994 (4). Ongoing cases of VAPP have been reported including case clusters (5), although there were no further cases of VAPP observed within the region that the child received the dose of OPV in this instance.

We describe a case that initially was consistent with the diagnosis of GBS. Few MRI scans are performed early in the course of polio infection. From the evolution of disease in our patient, it can be demonstrated that he progressed to develop poliomyelitis.

In considering the differential diagnosis, other possibilities would include GBS, nonpolio enteroviral infection, West Nile Virus infection, wild poliovirus infection and, finally, VAPP. GBS is an unlikely diagnosis given that the child had developed persistent one-limb weakness and these deficits persisted despite appropriate therapy for GBS. Nonpolio enteroviral infections were a possibility; however, they were ruled out by two stool cultures performed at the time of onset of clinical illness and both were negative. West Nile virus is observed in the area of Canada where the child lived, but his serology (immunoglobulin M) was negative. The diagnosis of wild poliovirus was discarded because the polio strain isolated from the child's stool showed 99.7\% homology with the strain used in OPV.

Further clinical, laboratory and radiological findings made this a probable case of VAPP. This case was unusual because the child appeared to be immunocompetent and had received two previous doses of IPV. In the United States, during the transition from an allOPV schedule to a mixed OPV/IPV schedule, no cases of VAPP associated with the mixed schedule were reported (6). All cases of VAPP reported in the United States were in children who received the OPV vaccine. The estimates of risk for VAPP range from one in 750,000 with the first dose of OPV to one in 2.4 million for all doses of OPV $(6,7)$. From the literature, it is evident that two doses of IPV provide high levels of protection (>90\%) for poliovirus 1 and 2 , but only $75 \%$ protection against poliovirus 3 (8).

The issue of OPV and its ability to cause VAPP has been intensely debated in countries in which the ability to maintain cold chains and other logistical as well as financial issues has continued to be debated (9). As the international efforts for polio eradication move forward, issues such as the present case arise as discussion points.

\section{REFERENCES}

1. Varughese P, Carter A, Acres S, Furesz J. Eradication of indigenous poliomyelitis in Canada: Impact of immunization strategies. Can J Public Health 1989;80:363-8.

2. Certification of poliomyelitis eradication - the Americas, 1994. MMWR 1994;43:720-2.

3. National Advisory Committee on Immunization. Canadian Immunization Guide, 7th edn. Ottawa: Public Health Agency of Canada, 2006.

4. Certification of poliomyelitis eradication - Western Pacific Region, October, 2000. MMWR 2001;50:1-3.

5. Liang X, Zhang Y, Xu W, et al. An outbreak of poliomyelitis caused by type 1 vaccine-derived poliovirus in China. 2006;194:545-51.

6. Alexander L, Seward J, Santibanez T, et al. Vaccine policy changes and epidemiology of poliomyelitis in the United States. JAMA 2004;292:1696-701.

7. Marx A, Glass J, Sutter R. Differential diagnosis of acute flaccid paralysis and its role in poliomyelitis surveillance. Epidemiol Rev 2000;22:298-316.

8. Modlin JF, Halsey NA, Thoms ML, Meschievitz CK, Patriarca PA. Humoral and mucosal immunity in infants induced by three sequential inactivated poliovirus vaccine-live attenuated oral poliovirus vaccine immunization schedules. Baltimore Area Polio Vaccine Study Group. J Infect Dis 1997;175(Suppl 34):S228-34.

9. John TJ. A developing country perspective on vaccine-associated paralytic poliomyelitis. Bull World Health Organ 2004;82:53-7. 


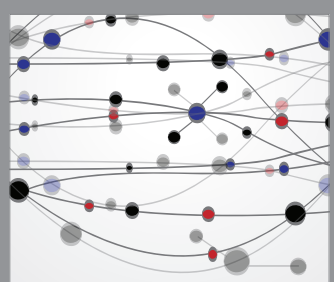

The Scientific World Journal
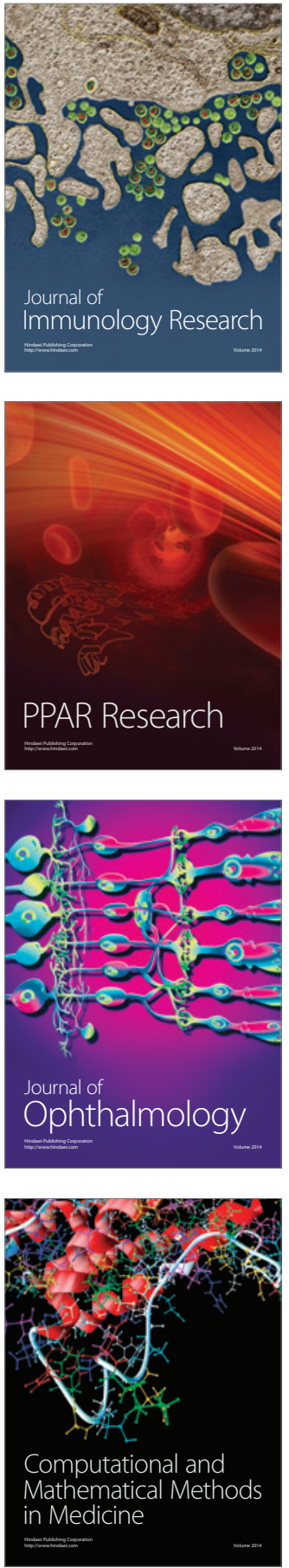

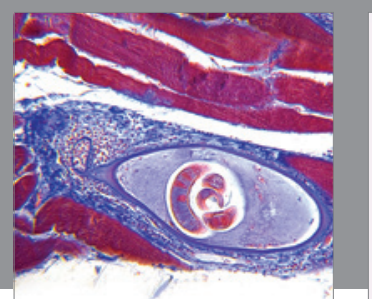

Gastroenterology Research and Practice

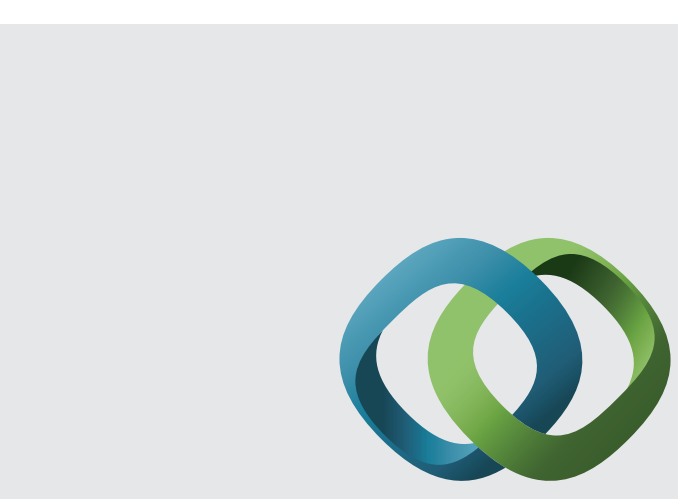

\section{Hindawi}

Submit your manuscripts at

http://www.hindawi.com
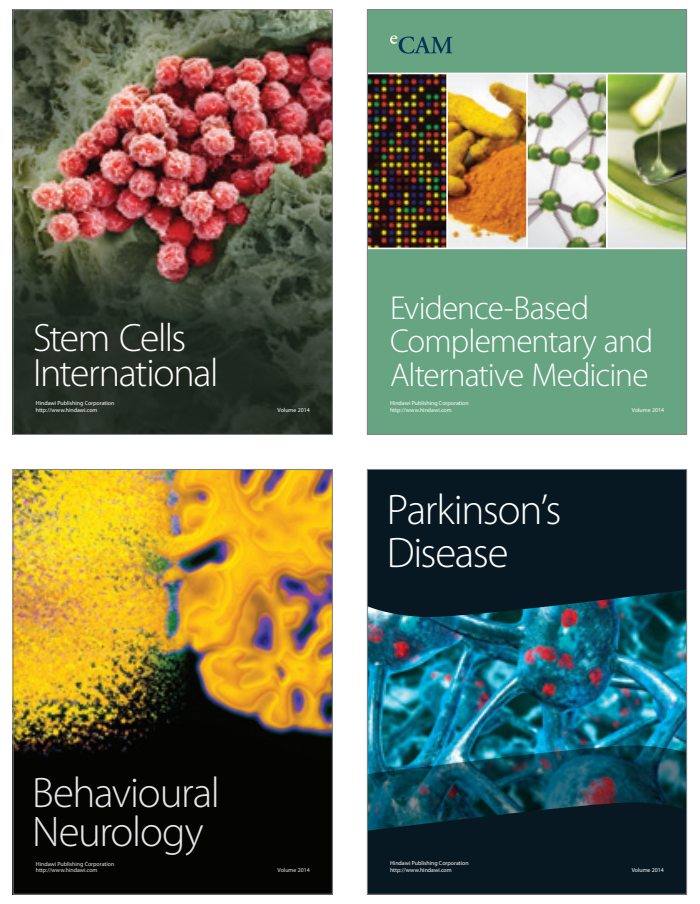
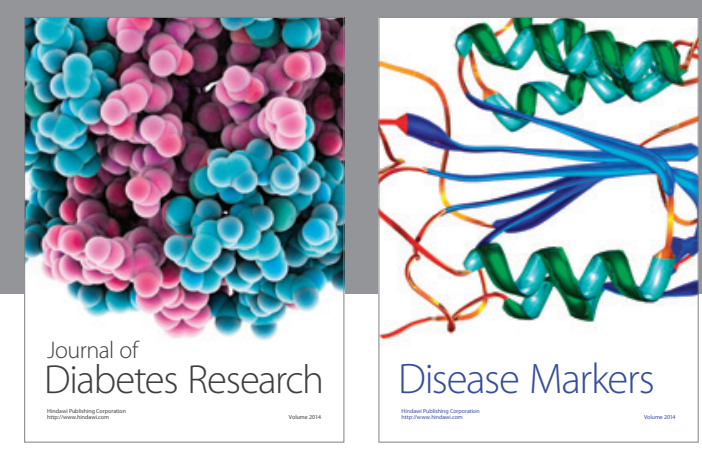

Disease Markers
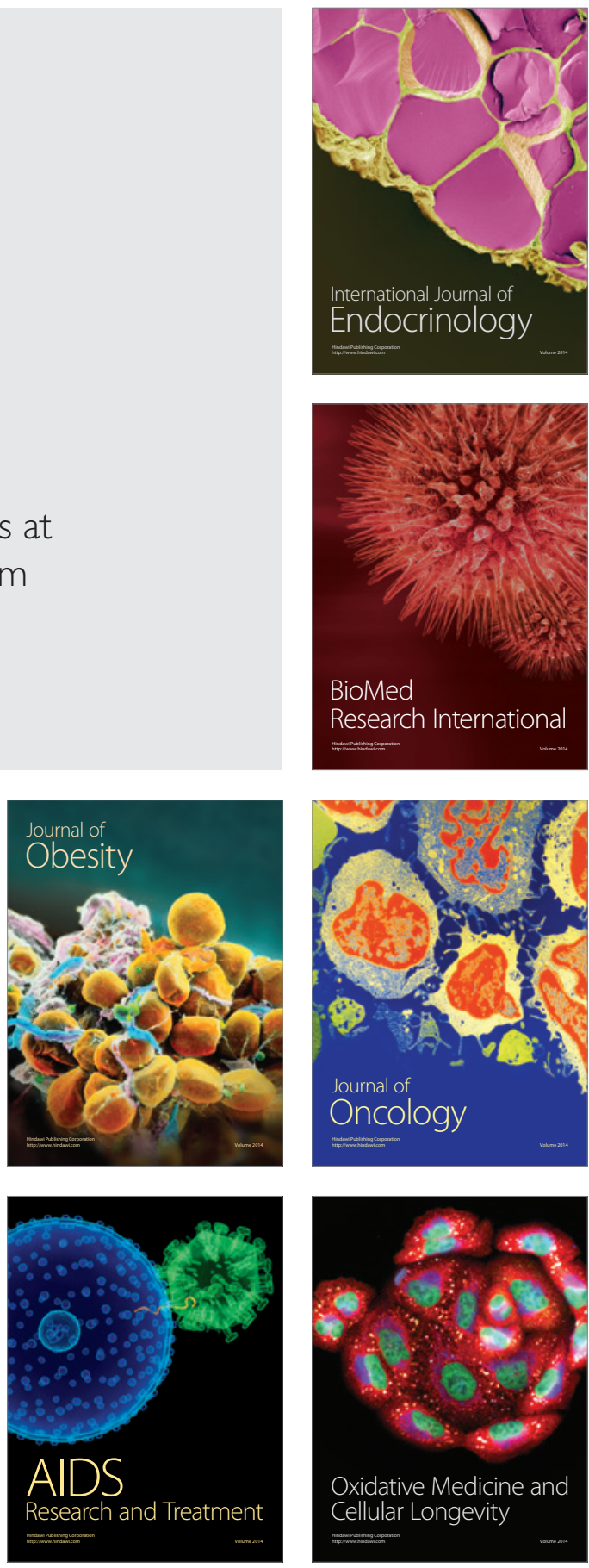